\title{
A Rare Case of Severe Muscular Necrosis Due to Extravascular Leakage of Trabectedin
}

-Severe Tissue Damage of Trabectedin Extravasation

\author{
Reika Aoyama1, Atsushi Tanemura1, Madoka Takafuji' ${ }^{1}$, Yorihisa Kotobuki', Aya Tanaka1, \\ Ichiro Katayama1, Satoshi Takenaka²
}

${ }^{1}$ Department of Dermatology, Graduate School of Medicine, Osaka University, Osaka, Japan

${ }^{2}$ Department of Orthopedics, Graduate School of Medicine, Osaka University, Osaka, Japan

Email: tanemura@derma.med.osaka-u.ac.jp

How to cite this paper: Aoyama, R., Tanemura, A., Takafuji, M., Kotobuki, Y., Tanaka, A., Katayama, I. and Takenaka, S. (2018) A Rare Case of Severe Muscular Necrosis Due to Extravascular Leakage of Trabectedin. Journal of Cosmetics, Dermatological Sciences and Applications, 8, 6-9.

https://doi.org/10.4236/jcdsa.2018.81002

Received: January 18, 2018

Accepted: March 5, 2018

Published: March 8, 2018

Copyright $\odot 2018$ by authors and Scientific Research Publishing Inc. This work is licensed under the Creative Commons Attribution International License (CC BY 4.0).

http://creativecommons.org/licenses/by/4.0/

\begin{abstract}
Trabectedin is a synthetic antineoplastic drug, binding to the minor groove of DNA and affecting DNA repair pathways, resulting in G2-M cell cycle arrest and apoptosis. Trabectedin has demonstrated high efficacy against various soft tissue sarcomas. However, its extravasation causes serious complications, such as tissue necrosis and a delay in the treatment of underlying diseases. Methods: We experienced a rare case in which trabectedin extravasation caused severe pectoralis major muscle necrosis. A 45-year-old man with multiple lung metastases of follicular dendritic cell sarcoma received $2.15 \mathrm{mg}$ of trabectedin totally through a central venous access device (CVAD) system in the right precordium. Computed tomography showed extensive turbidity of subcutaneous fatty tissue and swelling of the pectoralis major muscle to the upper margin of the liver, and the creatine kinase level was elevated to 759 $\mathrm{U} / \mathrm{L}$ (reference value from 54 to 286). We performed surgical debridement twice, and the CVAD was concomitantly removed; thereafter, the skin defect was reconstructed with a split skin mesh graft. Results: Histopathology showed extreme degeneration of striated muscle and fatty tissue. Unfortunately, disability of the right arm abducens persisted after treatment because of debridement around the right humerus muscle. Discussion: Several reports have described cases of the extravasation of trabectedin. A few have mentioned severe muscular degeneration similar to that shown in the present case. Because trabectedin is a strong vesicant cytotoxic agent, it is principally administered through a CVAD rather than peripheral vessels and is continued during the nighttime; this can lead to a delay in patients or attending doctors noticing any extravasation. We need to spread appropriate knowledge of this drug and make an effort to prevent severe complications like in
\end{abstract}


the present case.

\section{Keywords}

Trabectedin, Management of Extravasation, Vesicant Drug, Follicular Dendritic Cell Sarcoma, Muscular Involvement

\section{Introduction}

Trabectedin is a synthetic antineoplastic drug originally isolated from the Caribbean sea squirt. It binds to the minor groove of DNA, forming adducts and resulting in bending of the DNA helix towards the major groove. Adduct formation triggers a cascade of events that can affect DNA repair pathways, resulting in G2-M cell cycle arrest and apoptosis [1] - a novel anti-cancer mechanism. Trabectedin has shown high anti-tumor efficacy against various soft tissue sarcomas, with a disease control rate of $66.7 \%$ [1], and thus it was recently approved by the Japanese government for the treatment of soft tissue malignancies. However, its extravasation occasionally induces serious complication of surrounding tissue necrosis followed by the delayed treatment of underlying diseases.

\section{Methods}

We experienced a case of trabectedin extravasation that induced severe pectoralis major muscle necrosis. A 45-year-old-man with multiple lung metastases of follicular dendritic cell sarcoma first received almost full dose of trabectedin (2.15 $\mathrm{mg})$ through a central venous access device (CVAD) system in the right precordium. He noticed swelling and slight pain of his right frontal chest $21 \mathrm{~h}$ after the administration. Erythema and tenderness from the third day were exacerbated, so we suspected extravasation of trabectedin. We administered a subcutaneous injection of triamcinolone acetonide, which was not effective. Computed tomography revealed extensive turbidity of subcutaneous fatty tissue and swelling of the pectoralis major muscle to the upper margin of the liver (Figure 1(a)). The creatine kinase level was elevated to $759 \mathrm{U} / \mathrm{L}$. We performed surgical debridement twice, and removed the CVAD concomitantly; thereafter, skin defect was reconstructed with a split skin mesh graft (Figure 1(b)). We have received an agreement from this patient after informed consent for paper preparation.

\section{Results}

Histopathology revealed extreme degeneration of the striated muscle and fatty tissue (Figure 1(c), Figure 1(d)). Although grafted skin was well engrafted and epithelized, abduction failure of the right arm remained due to intense debridement and skin contracture around the right humerus muscle (Figure 1(e)). 
(a)

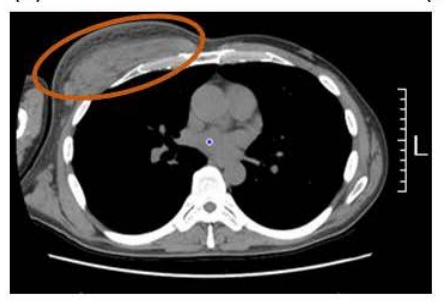

(d) (b)

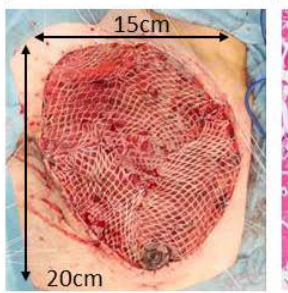

(e) (c)

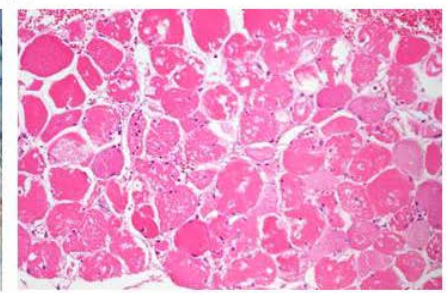

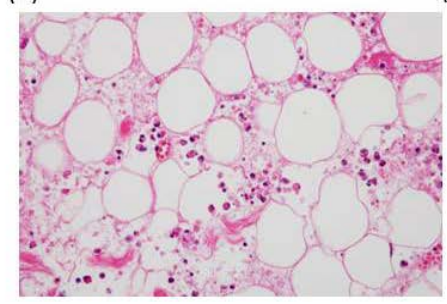

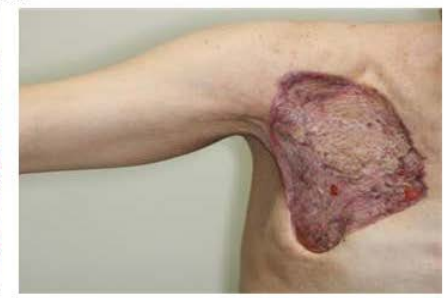

Figure 1. (a) Chest CT revealed extensive turbidity of subcutaneous fatty tissue and swelling of the pectoralis major muscle to the upper margin of liver. The red circle indicates turbid subcutaneous fatty tissue and swollen muscle; (b) The skin defect was reconstructed by a split skin mesh graft after surgical debridement and the removal of the CVAD; (c) (d) Histopathological findings showed extreme degeneration of striated muscle (HE, $\times 200)$ and overlying fatty tissue (HE, $\times 400$ ); (e) The patient suffered from disability of the right arm abducens because of debridement around the right humerus muscle.

Since trabectedin was not effective for lung metastatic lesions, we switched to administration of eribulin mesilate as alternative treatment.

\section{Discussion}

Extravasation from a CVAD has occurred in $0.3 \%-4.7 \%$ of cases [2]. Pre-marketing surveillance showed that $0.4 \%$ of patients (i.e. 4 of 950 cases) suffered from tissue injury caused by extravasation of trabectedin (unpublished data). Extravasation of trabectedin has been reported in seven cases [2] [3] [4] [5] since Theman et al. first described it in 2009 [3]. A few reports have also shown intense muscular degeneration similar to the present case [2] [3] [5]. To our knowledge, this is the one that induced tissue degeneration of pectralis major muscle more severely compared to previous reports.

Because trabectedin is a strong vesicant cytotoxic agent, it is principally administered through a CVAD rather than peripheral vessels and is continued during the nighttime; this can lead to a delay in patients or attending doctors noticing any extravasation. Several reasons for extravascular leakage, such as CVAD and/or infusion needle trouble, have been suggested. Extravasation in the present case may have been due to insufficient insertion of the infusion needle not reaching CVAD. No effective method of treating extravasation of trabectedin has yet been determined, aside from curable debridement of damaged soft tissue. To prevent severe complications observed in the present case, medical staff must be instilled with the appropriate pharmacological knowledge regarding trabectedin and its risk of extravasation, and the infusion must be aborted as 
soon as patients notice local signs of incompatibility.

\section{Conclusion}

We reported a rare case of severe muscular necrosis due to extravascular leakage of trabectedin. Newly developed trabectedin has clinical data which indicates high efficiency against various soft tissue sarcomas, but may cause serious cutaneous complication like the present case. We must share a proper knowledge of this drug with both of patients and medical stuff.

\section{References}

[1] Le Cesne, A., Ray-Coquard, I., Duffaud, F., et al. (2015) Trabectedin in Patients with Advanced Soft Tissue Sarcoma: A Retrospective National Analysis of French Sarcoma Group. European Journal of Cancer, 51, 742-750.

https://doi.org/10.1016/j.ejca.2015.01.006

[2] Haslik, W., Hacker, S., Felberbaucer, F.X., et al. (2015) Port-a-Cath Extravasation of Vesicant Cytotoxics: Surgical Options for a Rare Complication of Cancer Chemotherapy. European Journal of Surgical Oncology, 41, 378-385.

https://doi.org/10.1016/j.ejso.2014.11.042

[3] Theman, T.A., Hartzwll, T.L., Sinha, I., et al. (2009) Recognition of a New Chemotherapeutic Vesicant: Trabectedin (Ecteinascidin-743) Extravasation with Skin and Soft Tissue Damage. Journal of Clinical Oncology, 27, e198-e200. https://doi.org/10.1200/JCO.2008.21.6473

[4] Schoffski, P., Cerbone, L., Wolter, P., et al. (2012) Administration of 24-h Intravenous Infusions of Trabectedin in Ambulatory Patients with Mesenchymal Tumors via Disposable Elastomeric Pumps; An Effective and Patient-Friendly Palliative Treatment Option. Onkologie, 35, 14-17. https://doi.org/10.1159/000335879

[5] Yoshimi, K., Otsuka, M., Goto, H., Katagiri, H. and Hosaka, S. (2017) Differential Diagnosis of Trabectedin Extravasation: A Case Report. The Journal of Dermatolo$g y$, 44, e200-e201. https://doi.org/10.1111/1346-8138.13854 